\title{
ALTERAÇÃO ESTRUTURAL DE ÁREAS DE FLORESTAS EXPLORADAS CONVENCIONALMENTE EM PLANOS DE MANEJO, NOS DOMÍNIOS DE FLORESTA ATLÂNTICA, MINAS GERAIS, BRASIL ${ }^{1}$
}

\author{
Danilo José da Silva Coelho², Agostinho Lopes de Souza ${ }^{3}$, Carlos Pedro Boechat Soares ${ }^{3}$, João Augusto
} Alves Meira Neto ${ }^{4}$ e Helio Garcia Leite ${ }^{3}$

\begin{abstract}
RESUMO - Este estudo teve como objetivo analisar as alterações nas estruturas horizontal, diamétrica e interna em áreas de planos de manejo florestal exploradas convencionalmente. Utilizaram-se indicadores e verificadores, tendo como testemunha a área de reserva legal. Decorrido o tempo de exploração florestal de cada Plano de Manejo Florestal (PMF) e comparando as áreas de manejo florestal e áreas de reserva legal, com relação a alterações na estrutura horizontal dos PMFs, o verificador densidade absoluta indicou que as áreas de manejo florestal foram iguais estatisticamente $(\mathrm{P}>0,05)$ às áreas de reserva legal nos $\mathrm{PMF}_{28}, \mathrm{PMF}_{29}$ e $\mathrm{PMF}_{30}$ e diferentes $(\mathrm{P} \leq 0,05)$ no $\mathrm{PMF}_{16}$. Os verificadores dominância absoluta e volume total com casca indicaram que os estoques de área basal e volume total das áreas de manejo florestal foram iguais estatisticamente $(\mathrm{P}>0,05)$ às áreas de reserva legal nos $\mathrm{PMF}_{28}, \mathrm{PMF}_{30}$ e $\mathrm{PMF}_{16}$ e diferentes $(\mathrm{P} \leq 0,05)$ no $\mathrm{PMF}_{29}$. Com relação a alterações na estrutura diamétrica dos PMFs, os verificadores distribuição da densidade absoluta, área basal e volume total com casca, por hectare e por classe diamétrica, indicaram que as áreas de manejo florestal foram iguais estatisticamente $(\mathrm{P}>0,05)$ às áreas de reserva legal nos $\mathrm{PMF}_{28}, \mathrm{PMF}_{29}$ e $\mathrm{PMF}_{30}$ e diferentes $(\mathrm{P} \leq 0,05)$ no $\mathrm{PMF}_{16}$. Com relação às alterações na estrutura interna dos PMFs, o verificador infestação de cipós indicou que as áreas de manejo florestal apresentaram infestação de cipós igual estatisticamente $(\mathrm{P}>0,05)$ às áreas de reserva legal nos $\mathrm{PMF}_{29}$, $\mathrm{PMF}_{30}$ e $\mathrm{PMF}_{16}$ e diferente $(\mathrm{P} \leq 0,05)$ no $\mathrm{PMF}_{28}$. O verificador qualidade de fuste mostrou que as áreas de manejo florestal possuem qualidade de fuste igual estatisticamente $(\mathrm{P}>0,05)$ às áreas de reserva legal em todos os PMFs.
\end{abstract}

Palavras-chave: Floresta estacional semidecidual, floresta atlântica e alteração.

\section{STRUCTURAL CHANGES IN CONVENTIONALLY EXPLOITED FOREST AREAS IN MANAGEMENT PLANS, IN THE ATLANTIC FLOREST DOMAIN, MINAS GERAIS STATE, BRAZIL}

\begin{abstract}
The objective of the present study was to analyze the changes in the horizontal, diametric and internal structures in conventionally exploited areas of forest management plans. Indicators and verifiers were used having as control the legal reserve area. After the forest harvest period of each FMP and comparing the forest management areas with the legal reserve areas with respect to changes in the horizontal structure of the FMPs, the verifier absolute density indicated that the forest management areas were statistically equal $(p>0.05)$ to legal reserve areas in the $F M P_{28}, F M P_{29}$ and $F M P_{30}$ and different $(P=0.05)$ in the $F M P_{16}$. The verifiers outside absolute dominance and total volume outside bark indicated that the stocks of basal area and total volume of the forest management areas were statistically equal $(P>0.05)$ to legal reserve areas in the $F M P_{28}, F M P_{30}$ and $F M P_{16}$, and different $(P=0.05)$ in the $F M P_{29}$. In relation to changes in the diametric structure of PMFs, the verifiers distribution of absolute density, basal area and outside bark volume, per
\end{abstract}

\footnotetext{
${ }^{1}$ Recebido em 24.04.2006 e aceito para publicação em 14.05.2007.

${ }^{2}$ Instituto Estadual de Florestas (IEF), Cpmaf Centro de Pesquisa de Manejo Florestal. E-mail: <djcoelho@ufv.br>.

${ }^{3}$ Departamento de Engenharia Florestal da Universidade Federal de Viçosa (UFV). E-mail: <alsouza@ufv.br>.

${ }^{4}$ Departamento de Biologia Vegetal da Universidade Federal de Viçosa (UFV). E-mail: <j.meira@ufv.br>.
} 
hectare and per diametric class, indicated that the forest management areas were statistically equal $(P>0.05)$ to legal reserve areas in the $F M P_{28}, F M P_{29}$ and $F M P_{30}$, and different $(P=0.05)$ in the $F M P_{16}$. In relation to changes in the internal structure of the FMPs, the verifier infestation by lianes indicated that the forest management areas showed infestation by lianes statistically equal $(P>0.05)$ to legal reserve areas in the $F M P_{29}$, $F M P_{30}$ and $F M P_{16}$, and different $(P=0.05)$ in the $F M P_{28}$. The verifier log quality showed that the managed forest areas had log quality statistically equal $(P>0.05)$ to the legal reserve areas in all the FMPs.

Keywords: Semideciduous seasonal forest, Atlantic forest and structural change.

\section{INTRODUÇÃO}

No ano de 1991, Minas Gerais, por meio do Poder Legislativo do Estado e pela primeira vez na sua história, deu um tratamento específico às questões florestais, ordenando as atividades florestais através da publicação da Lei no 10.561, de 27 de dezembro de 1991. A partir de então, qualquer exploração florestal passou a depender de prévia autorização do órgão estadual competente, e a exploração de florestas nativas primárias ou em estágio médio ou avançado de regeneração, para fins de carvoejamento, aproveitamento industrial, comercial ou outros fins, em áreas de formações florestais, somente se dará através de plano de manejo florestal de rendimento sustentável.

O plano de manejo florestal de que trata a legislação deverá ser executado com o objetivo de prover o manejo ecológico das espécies e dos ecossistemas estaduais, assegurando um meio ambiente ecologicamente equilibrado.

O Instituto Estadual de Florestas (IEF-MG), órgão responsável pela gestão dos recursos florestais do Estado, começou, a partir daquela data, a protocolar planos de manejo para exploração florestal em várias regiões mineiras. Nas áreas de formações florestais foram protocolados, nos escritórios do IEF-MG, 132 planos no período de 1993 a 1998, sendo 70 planos $(53,07 \%)$ na microrregião da Zona da Mata, $60(45,46 \%)$ na microrregião Centro Sul e $2(1,31 \%)$ na microrregião Nordeste (CPMF, 2000). Desse total, 78 planos $(58,2 \%)$ possuem áreas de exploração de 10 ha, 33 (24,62\%) entre 10 e 20 hae $22(16,42 \%)$ acima de 50 ha, caracterizando o manejo em pequenos fragmentos florestais, o que dificulta, sobremaneira, a sua fiscalização e o seu monitoramento.

Até hoje, nenhum trabalho foi executado para se conhecer a real situação técnica de planos protocolados, autorizados e executados ou em execução. É extremamente importante avaliar a qualidade técnica desses planos, tendo em vista a sua sustentabilidade biológica. Assim, este trabalho teve como objetivo analisar, por meio de indicadores e verificadores, as alterações nas estruturas horizontal, interna e diamétrica de planos de manejo florestal (PMFs) previamente selecionados e representativos da população dos PMFS autorizados no Estado de Minas Gerais.

\section{MATERIAL E MÉTODOS}

\section{1. Área de estudo}

O estudo foi realizado nas fazendas Amoras $\left(\mathrm{PMF}_{28}\right)$, Maia-Vassorouras $\left(\mathrm{PMF}_{29}\right)$, Retiro $\left(\mathrm{PMF}_{30}\right)$ e Alves $\left(\mathrm{PMF}_{16}\right)$, nos Municípios de Porto Firme e Rio Espera, respectivamente, localizados na mesorregião Zona da Mata, no Sudeste de Minas Gerais, entre as coordenadas geográficas $19^{\circ} 45^{\prime}$ e $22^{\circ} 15^{\prime}$ de latitude sul e $41^{\circ} 15^{\prime}$ e $44^{\circ} 15^{\prime}$ de longitude oeste.

A região apresenta relevo plano (7\%), ondulado (21\%) e montanhoso (72\%) com característica fortemente de ondulado e montanhoso, com encostas de perfil convexo-côncavo embutido em vales de fundo chato, formados por terraços e leitos maiores, onde ocorrem cursos d'água. A temperatura média anual oscila em torno de $18,5^{\circ} \mathrm{C}$, e o índice médio pluviométrico anual é de aproximadamente $1.380 \mathrm{~mm}$ (MINAS GERAIS, 2005).

As formações florestais incluem-se no domínio da floresta Atlântica (Floresta Estacional Semidecidual) (VELOSO, 1992). Seus agrupamentos remanescentes mais expressivos localizam-se nas encostas superiores dos terrenos da região. Ainda que variada quanto à estrutura e à composição, destacam-se, quanto à flora, as espécies Copaifera langsdorffii Desf. (copaíba), Ocotea sp. e Nectandra sp. (Canelas), Schizolobium parahyba (Vell.) Blake (guapuruvu), Cedrela fissilis 
Dell. (cedro), Plathymenia foliolosa Benth. (vinhático), Aspidosperma polyneuron M. Arg. (peroba-rosa) e Cariniana legalis (Mart.) Kuntze (jequitibá-rosa). Outras extensões da Floresta Estacional Semidecidual na região são as galerias ao longo dos cursos d'água (floresta de galeria, mata ciliar), ocupando áreas em condições de umidade permanente (IEF, 1994).

\subsection{Amostragem}

O PMF ${ }_{28}$ (10 anos após a exploração florestal), $\mathrm{PMF}_{29}$ (8 anos após a exploração florestal), $\mathrm{PMF}_{30}(9$ anos após a exploração florestal) e $\mathrm{PMF}_{16}$ (11 anos após a exploração florestal) foram selecionados previamente, após análise de agrupamento (utilizandose a distância euclidiana simples e o método de Ward), em função da área basal, de todos os planos protocolados na microrregião da Zona da Mata de Minas Gerais. Visando verificar as alterações na estrutura das florestas após o período de exploração de cada plano, foram selecionadas 20 parcelas de área fixa de $500 \mathrm{~m}^{2}$ (10 x $50 \mathrm{~m}$ ), por PMF, sendo 10 parcelas em área de manejo florestal e 10 em área de reserva legal. As parcelas foram distribuídas de modo a contemplar o gradiente de declividade ao longo do terreno (Figura 1). Todos os indivíduos vivos ou mortos em pé, com CAP (circunferência a 1,30 m do solo) igual ou superior a $15 \mathrm{~cm}$, foram medidos, bem como estimadas a altura total e a comercial, coletando-se o material botânico para identificação.

\subsection{Análise estatística}

Para a análise estatística, foram admitidos dois tratamentos (área de manejo florestal e área de reserva legal), com 10 repetições cada. Com relação às variáveis em estudo, foram aplicados testes de normalidade de Lillierfors (LILIEFORS, 1967) e os de homogeneidade das variâncias de Cochran e Balrllet (WINER, 1962).

Os resultados obtidos a partir dos levantamentos florísticos e estruturais das áreas de manejo florestal e áreas de reserva legal foram submetidos ao teste de $\mathrm{t}$, de Student, a 5\% de probabilidade, para verificar a existência de alterações significativas entre as médias dos verificadores analisados.

Para comparar as distribuições de densidade (n $\left.\mathrm{ha}^{-1}\right)$, área basal $\left(\mathrm{m}^{2} \mathrm{ha}^{-1}\right)$ e volume total $\left(\mathrm{m}^{3} \mathrm{ha}^{-1}\right)$, por classe de diâmetro, entre as áreas de manejo florestal e de reserva legal, utilizou-se o teste L \& O, proposto por Leite e Oliveira (2002), que é uma combinação do teste $F$ modificado de Graybill, teste $t$ para o erro médio, e da análise do coeficiente de correlação linear.

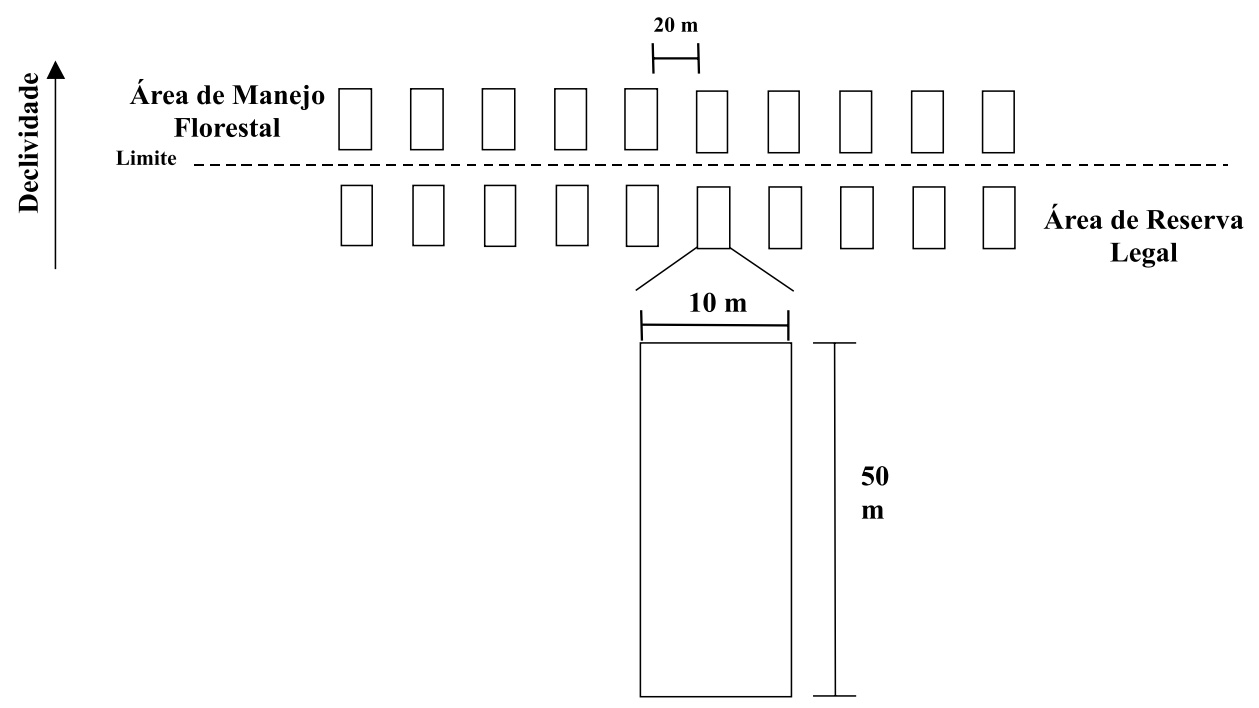

Vegetação arbórea: $\operatorname{dap} \geq 5 \mathrm{~cm}$

Figura 1 - Desenho do sistema de amostragem executado nos planos de manejo florestal selecionados com base na análise de agrupamento, para serem inventariados no campo.

Figure 1 -Drawing of the sampling system performed in the forest management plans selected based on the cluster analysis, to be surveyed in the field. 
O volume total com casca das árvores individuais foi estimado pela seguinte equação do CETEC (1995), ajustada para floresta secundária:

$$
\begin{aligned}
& \text { Vtcc }=0,000074230 \mathrm{Dap}^{1,707348} \mathrm{Ht}^{1,16873} \\
& \mathrm{R}^{2}=93,30 \%
\end{aligned}
$$

Os dados de campo das parcelas foram processados utilizando-se o programa MATANATIVA 2.0 (CIENTEC, 2006) e as análises estatísticas, executadas pelo software SAEG (2005).

\section{RESULTADOS E DISCUSSÃO}

\subsection{Alterações na estrutura horizontal dos PMFs}

\subsubsection{Densidade absoluta}

Para o nível de inclusão de $5 \mathrm{~cm}$ de DAP, no $\mathrm{PMF}_{28}$, a área de manejo florestal (Quadro 1) apresentou 1.500 indivíduos por hectare ( $\left.\mathrm{n} \mathrm{ha}^{-1}\right)$, em que cinco espécies seguintes de maior densidade absoluta contribuíram com 34,48\% dos indivíduos: Mabea fistulifera Mart. (180 n ha $\left.^{-1}\right)$, Apuleia leiocarpa J.F. Macbr. (142 $\left.\mathrm{n} \mathrm{ha}^{-1}\right)$, Duguetia sp. $\left(74 \mathrm{n} \mathrm{ha}^{-1}\right)$, Myrciafallax DC. $\left(70 \mathrm{n} \mathrm{ha}^{-1}\right)$ e Carpotroche brasiliensis Endl. (56 $\mathrm{n} \mathrm{ha}^{-1}$ ). Na área de reserva legal, a densidade total foi de $1.468 \mathrm{n} \mathrm{ha}^{-1}$, e as cinco espécies seguintes de maior densidade absoluta contribuíram com 37,49\% dos indivíduos: Maprounea guianensis Aubl. (164 $\left.\mathrm{n} \mathrm{ha}^{-1}\right)$, Xylopia brasiliensis Spreng. (122 $\left.\mathrm{n} \mathrm{ha}^{-1}\right)$, Casearia decandra Jacq. (106 $\mathrm{n} \mathrm{ha}^{-1}$ ), Mabea fistulifera (92 $\mathrm{n} \mathrm{ha}^{-1}$ ) e Lacistema pubescens Mart. (66 $\mathrm{n} \mathrm{ha}^{-1}$ ).

No $\mathrm{PMF}_{29}$, a área de manejo florestal apresentou $1.880 \mathrm{n} \mathrm{ha}^{-1}$, e as cinco espécies seguintes de maior densidade absoluta contribuíram com 60,42\% dos indivíduos: Lacistema pubescens (328 $\left.\mathrm{n} \mathrm{ha}^{-1}\right)$, Mabea fistulifera $\left(284 \mathrm{n} \mathrm{ha}^{-1}\right)$, Casearia decandra (196 n ha $\left.{ }^{-1}\right)$, Rollinia silvatica Mart. (174 $\left.\mathrm{n} \mathrm{ha}^{-1}\right)$ e Xylopia brasiliensis $\left(154 \mathrm{n} \mathrm{ha}^{-1}\right)$. Na área de reserva legal, a densidade total foi de $1.946 \mathrm{n} \mathrm{ha}^{-1}$, com a ressalva de que as cinco espécies seguintes de maior densidade absoluta contribuíram com 47,58\% dos indivíduos: Mabea fistulifera (238 $\left.\mathrm{n} \mathrm{ha}^{-1}\right)$, Xylopia brasiliensis $\left(184 \mathrm{nha}^{-1}\right)$, Rollinia silvatica $\left(180 \mathrm{nha}^{-1}\right)$, Lacistema pubescens (168 $\left.\mathrm{n} \mathrm{ha}^{-1}\right)$ e Casearia decandra (156 n ha-1).

No $\mathrm{PMF}_{30}$, a área de manejo florestal apresentou $1.488 \mathrm{n} \mathrm{ha}^{-1}$, e as cinco espécies seguintes de maior densidade absoluta contribuíram com 62,63\% dos indivíduos: Mabea fistulifera (534 $\left.\mathrm{n} \mathrm{ha}^{-1}\right)$, Apuleia leiocarpa (134 $\left.\mathrm{n} \mathrm{ha}^{-1}\right)$, Xylopia sericea A. St.-Hil. (114 $\left.\mathrm{n} \mathrm{ha}^{-1}\right)$, Matayba elaeagnoides Radlk. (94 $\mathrm{n} \mathrm{ha}^{-1}$ ) e Lacistema pubescens (56 $\left.\mathrm{n} \mathrm{ha}^{-1}\right)$. Na área de reserva legal, a densidade total foi de $1.524 \mathrm{n} \mathrm{ha}^{-1}$, ressaltando-se que as cinco espécies seguintes de maior densidade absoluta contribuíram com 44,22\% dos indivíduos: Xylopia sericea (302 $\left.\mathrm{n} \mathrm{ha}^{-1}\right)$, Apuleia leiocarpa (118 $\left.\mathrm{n} \mathrm{ha}^{-1}\right)$, Lacistema pubescens $\left(92 \mathrm{n} \mathrm{ha}^{-1}\right)$, Matayba elaeagnoides ( $82 \mathrm{n} \mathrm{ha}^{-1}$ ) e Mabea fistulifera $\left(80 \mathrm{nha}^{-1}\right)$.

No $\mathrm{PMF}_{16}$, a área de manejo florestal apresentou $2.162 \mathrm{n} \mathrm{ha}^{-1}$, onde as cinco espécies seguintes de maior densidade absoluta contribuíram com 35,89\% dos indivíduos: Mabea fistulifera $\left(222 \mathrm{n} \mathrm{ha}^{-1}\right)$, Cecropia glaziovi Snethlage (156 n ha ${ }^{-1}$ ), Croton floribundus Spreng. (156 n ha-1), Jacaranda puberula Charm. (144 $\left.\mathrm{n} \mathrm{ha}^{-1}\right)$ e Aparisthmium cordatum Baill. (98 $\mathrm{n} \mathrm{ha}^{-1}$ ). Na área de reserva legal, a densidade total foi de $1.508 \mathrm{n} \mathrm{ha}^{-1}$, e as cinco espécies seguintes de maior densidade absoluta contribuíram com 24,40\% dos indivíduos: Xylopia brasiliensis (102 $\left.\mathrm{n} \mathrm{ha}^{-1}\right)$, Anadenanthera peregrina Speg. (74 $\left.\mathrm{n} \mathrm{ha}^{-1}\right)$, Mabea fistulifera $\left(74 \mathrm{n} \mathrm{ha}^{-1}\right)$, Casearia decandra $\left(60 \mathrm{n} \mathrm{ha}^{-1}\right) \mathrm{e}$ Casearia ulmifolia Cambess. (58 $\mathrm{n} \mathrm{ha}^{-1}$ ). Decorrido o tempo de 10 anos da exploração florestal do $\mathrm{PMF}_{28}$ oito anos do $\mathrm{PMF}_{29}$, nove anos do $\mathrm{PMF}_{30}$ e 11 anos do $\mathrm{PMF}_{16}$, o verificador densidade absoluta indicou que as áreas de manejo florestal são iguais, estatisticamente $(P>0,05)$, às áreas de reserva legal no $\mathrm{PMF}_{28}, \mathrm{PMF}_{29}$ e $\mathrm{PMF}_{30} \mathrm{e}$ diferentes $(\mathrm{P}=0,05)$ no $\mathrm{PMF}_{16}$.

Quadro 1 - Estimativa da densidade absoluta $\left(\mathrm{n} \mathrm{ha}^{-1}\right)$, dominância absoluta $\left(\mathrm{m}^{2} \mathrm{ha}^{-1}\right)$ e volume total com casca $\left(\mathrm{m}^{3} \mathrm{ha}^{-1}\right)$, em áreas de manejo florestal e áreas de reserva legal dos $\mathrm{PMF}_{28}, \mathrm{PMF}_{29}, \mathrm{PMF}_{30}$ e $\mathrm{PMF}_{16}$, nos municípios de Porto Firme e Rio Espera, Minas Gerais, em 2005

Table 1 - Estimations of absolute density $\left(n h a^{-1}\right)$, absolute dominance $\left(\left(m^{2} h a^{-1}\right)\right.$ and total volume outside bark $\left(m^{3} h a^{-1}\right)$, in forest management areas and legal reserve areas of the $F M P_{28}, F M P_{29}, F M P_{30}$ and $F M P_{16}$, in the municipalities of

\begin{tabular}{|c|c|c|c|c|c|c|c|c|}
\hline & \multicolumn{8}{|c|}{ Condição de Floresta } \\
\hline & \multicolumn{4}{|c|}{ AMF } & \multicolumn{4}{|c|}{$\overline{\mathrm{ARL}}$} \\
\hline & $\mathrm{PMF}_{28}$ & $\mathrm{PMF}_{29}$ & $\mathrm{PMF}_{30}$ & $\mathrm{PMF}_{16}$ & $\mathrm{PMF}_{28}$ & $\mathrm{PMF}_{29}$ & $\mathrm{PMF}_{30}$ & $\mathrm{PMF}_{16}$ \\
\hline$\overline{\text { Densidade }\left(\mathrm{n} \mathrm{ha}^{-1}\right)}$ & $1500^{\mathrm{ns}}$ & $1880^{\text {ns }}$ & $1488^{\mathrm{ns}}$ & $2162^{*}$ & 1468 & 1946 & 1524 & 1508 \\
\hline Dominância $\left(\mathrm{m}^{2} \mathrm{ha}^{-1}\right)$ & $14,283^{\mathrm{ns}}$ & $7,430^{*}$ & $9,996^{\mathrm{ns}}$ & $16,678^{\mathrm{ns}}$ & 17,562 & 10,160 & 9,875 & 17,208 \\
\hline Volume total $\left(\mathrm{m}^{3} \mathrm{ha}^{-1}\right)$ & $94,71^{\mathrm{ns}}$ & $37,07^{*}$ & $53,02^{\text {ns }}$ & $102,61^{\mathrm{ns}}$ & 115,00 & 54,69 & 49,61 & 95,03 \\
\hline
\end{tabular}
Porto Firme and Rio Espera, Minas Gerais State, in 2005

* Significativo a $5 \%$ e ns: não-significativo a $5 \%$.

R. Árvore, Viçosa-MG, v.31, n.5, p.867-877, 2007 


\subsubsection{Dominância absoluta}

No $\mathrm{PMF}_{28}$ para o nível de inclusão de $\mathrm{DAP}=5 \mathrm{~cm}$, a área de manejo florestal apresentou um estoque de área basal de 14,283 $\mathrm{m}^{2} \mathrm{ha}^{-1}$, onde as cinco espécies seguintes de maior área basal por hectare representaram 39,45\% do total: Anadenanthera peregrina $\left(1,943 \mathrm{~m}^{2} \mathrm{ha}^{-1}\right)$, Apuleia leiocarpa $\left(1,402 \mathrm{~m}^{2} \mathrm{ha}^{-1}\right)$, Mabea fistulifera $\left(0,926 \mathrm{~m}^{2} \mathrm{ha}^{-1}\right)$, Piptadenia gonoacantha J. F. Macbr. $\left(0,736 \mathrm{~m}^{2} \mathrm{ha}^{-1}\right)$ e Duguetia sp. $\left(0,629 \mathrm{~m}^{2} \mathrm{ha}^{-1}\right)$. Na área de reserva legal observou-se um estoque de $17,562 \mathrm{~m}^{2} \mathrm{ha}^{-1}$, onde as cinco espécies seguintes de maior área basal por hectare representaram 45,72\% do total: Xylopia brasiliensis $\left(2,132 \mathrm{~m}^{2} \mathrm{ha}^{-1}\right)$, Maprounea guianensis $\left(1,861 \mathrm{~m}^{2} \mathrm{ha}^{-1}\right)$, Ocotea $\mathrm{sp}$. (1,579 $\left.\mathrm{m}^{2} \mathrm{ha}^{-1}\right)$, Casearia decandra $\left(1,243 \mathrm{~m}^{2} \mathrm{ha}^{-1}\right)$ e morta $\left(1,215 \mathrm{~m}^{2} \mathrm{ha}^{-1}\right)$.

No $\mathrm{PMF}_{29}$, a área de manejo florestal apresentou um estoque de área basal de 7,430 $\mathrm{m}^{2} \mathrm{ha}^{-1}$, onde as cinco espécies seguintes de maior área basal representaram 57,44\% do total: Mabea fistulifera (1,078 $\left.\mathrm{m}^{2} \mathrm{ha}^{-1}\right)$, Lacistema pubescens $\left(1,072 \mathrm{~m}^{2} \mathrm{ha}^{-1}\right)$, Xylopia brasiliensis $\left(0,747 \mathrm{~m}^{2} \mathrm{ha}^{-1}\right)$, Rollinia silvatica $\left(0,727 \mathrm{~m}^{2} \mathrm{ha}^{-1}\right)$ e Miconia cinnamomifolia Naudin $\left(0,644 \mathrm{~m}^{2} \mathrm{ha}^{-1}\right)$. Na área de reserva legal, observou-se um estoque de $10,16 \mathrm{~m}^{2} \mathrm{ha}^{-1}$, onde as cinco espécies seguintes de maior área basal representavam $45,98 \%$ do total: Xylopia brasiliensis $\left(1,126 \mathrm{~m}^{2} \mathrm{ha}^{-1}\right)$, Mabea fistulifera $\left(1,114 \mathrm{~m}^{2} \mathrm{ha}^{-1}\right)$, Rollinia silvatica $\left(0,939 \mathrm{~m}^{2} \mathrm{ha}^{-1}\right)$, Casearia decandra $\left(0,769 \mathrm{~m}^{2} \mathrm{ha}^{-1}\right)$ e Lacistema pubescens $\left(0,724 \mathrm{~m}^{2} \mathrm{ha}^{-1}\right)$.

No $\mathrm{PMF}_{30}$ a área de manejo florestal apresentou um estoque de área basal de $9,996 \mathrm{~m}^{2}$ ha-1, onde as cinco espécies seguintes de maior área basal representavam 60,46\% do total: Mabea fistulifera $\left(3,269 \mathrm{~m}^{2} \mathrm{ha}^{-1}\right)$, Apuleia leiocarpa $\left(0,927 \mathrm{~m}^{2} \mathrm{ha}^{-1}\right)$, Matayba elaeagnoides $\left(0,742 \mathrm{~m}^{2} \mathrm{ha}^{-1}\right)$, Xylopia sericea (0,699 $\left.\mathrm{m}^{2} \mathrm{ha}^{-1}\right)$ e Hyptidendron asperrimum (Spreng.) Harley $\left(0,407 \mathrm{~m}^{2} \mathrm{ha}^{-1}\right)$. Na área de reserva legal, verificouse um estoque de $9,875 \mathrm{~m}^{2} \mathrm{ha}^{-1}$, onde as cinco espécies seguintes de maior área basal representavam 47,05\% do total: Xylopia sericea $\left(2,069 \mathrm{~m}^{2} \mathrm{ha}^{-1}\right)$, Apuleia leiocarpa $\left(0,886 \mathrm{~m}^{2} \mathrm{ha}^{-1}\right)$, Platymenia foliolosa Benth. $\left(0,777 \mathrm{~m}^{2} \mathrm{ha}^{-1}\right)$, Pitptocarpha macropoda Baker $\left(0,479 \mathrm{~m}^{2} \mathrm{ha}^{-1}\right)$ e Myrcia fallax $\left(0,435 \mathrm{~m}^{2} \mathrm{ha}^{-1}\right)$.

No $\mathrm{PMF}_{16}$ a área de manejo florestal apresentou um estoque de área basal de $16,678 \mathrm{~m}^{2} \mathrm{ha}^{-1}$, onde as cinco espécies seguintes de maior área basal representaram 34,07\% do total: Croton floribundus $\left(1,53 \mathrm{~m}^{2} \mathrm{ha}^{-1}\right)$, Cecropia glaziovi $\left(1,252 \mathrm{~m}^{2} \mathrm{ha}^{-1}\right)$, Mabea fistulifera $\left(1,152 \mathrm{~m}^{2} \mathrm{ha}^{-1}\right)$, Pitptocarpha macropoda $\left(0,946 \mathrm{~m}^{2} \mathrm{ha}^{-1}\right)$ e Platypodium elegans Vogel $\left(0,803 \mathrm{~m}^{2} \mathrm{ha}^{-1}\right)$. Na área de reserva legal, notou-se um estoque de área basal de $17,408 \mathrm{~m}^{2} \mathrm{ha}^{-1}$, onde as cinco espécies seguintes de maior área basal representavam 38,39\% do total: Xylopia brasiliensis (2,224 $\left.\mathrm{m}^{2} \mathrm{ha}^{-1}\right)$, Platypodium elegans $\left(1,615 \mathrm{~m}^{2} \mathrm{ha}^{-1}\right)$, Pseudopiptadenia contorta (DC.) G.P. Lewis \& M.P. Lima $\left(1,207 \mathrm{~m}^{2} \mathrm{ha}^{-1}\right)$, Anadenanthera peregrina $\left(0,831 \mathrm{~m}^{2} \mathrm{ha}^{-1}\right)$ e Dalbergia nigra Allemão ex Benth. $\left(0,807 \mathrm{~m}^{2} \mathrm{ha}^{-1}\right)$.

Decorrido o tempo de exploração florestal dos PMFs os verificadores dominância absoluta indicaram qué os estoques de área basal das áreas de manejo florestal eram iguais, estatisticamente $(\mathrm{P}>0,05)$, às áreas de reserva legal nos $\mathrm{PMF}_{28}, \mathrm{PMF}_{30}$, e $\mathrm{PMF}_{16}$ e diferentes, estatisticamente $(\mathrm{P}=0,05)$, no $\mathrm{PMF}_{29}$.

\subsubsection{Volume total com casca}

No $\mathrm{PMF}_{28}$, área de manejo florestal apresentou um volume total com casca de $97,71 \mathrm{~m}^{3} \mathrm{ha}^{-1}$, onde as cinco espécies seguintes representaram 47,71\% do volume total: Anadenanthera peregrina $\left(14,94 \mathrm{~m}^{3} \mathrm{ha}^{-1}\right)$, Apuleia leiocarpa $\left(10,90 \mathrm{~m}^{3} \mathrm{ha}^{-1}\right)$, Mabea fistulifera $\left(6,35 \mathrm{~m}^{3} \mathrm{ha}^{-1}\right)$, Piptadenia gonoacantha $\left(5,17 \mathrm{~m}^{3} \mathrm{ha}^{-1}\right)$ e Duguetia sp. $\left(4,37 \mathrm{~m}^{3}\right.$ $\left.\mathrm{ha}^{-1}\right)$. $\mathrm{Na}$ área de reserva legal, observou-se um estoque de volume total com casca de $76,34 \mathrm{~m}^{3} \mathrm{ha}^{-1}$, onde as cinco espécies seguintes representavam $65,99 \%$ do volume total: Xylopia brasiliensis $\left(15,04 \mathrm{~m}^{3} \mathrm{ha}^{-1}\right)$, Maprounea guianensis $\left(13,96 \mathrm{~m}^{3} \mathrm{ha}^{-1}\right)$, Ocotea $\mathrm{sp}$. $\left(11,19 \mathrm{~m}^{3} \mathrm{ha}^{-1}\right)$, Casearia decandra $\left(8,48 \mathrm{~m}^{3} \mathrm{ha}^{-1}\right) \mathrm{e}$ indeterminada $\left(6,56 \mathrm{~m}^{3} \mathrm{ha}^{-1}\right)$.

No $\mathrm{PMF}_{29}$, a área de manejo florestal apresentou um estoque de volume total de $37,07 \mathrm{~m}^{3} \mathrm{ha}^{-1}$, onde as cinco espécies seguintes representam $58,32 \%$ do volume total: Mabea fistulifera $\left(6,18 \mathrm{~m}^{3} \mathrm{ha}^{-1}\right)$, Lacistema pubescens $\left(4,61 \mathrm{~m}^{3} \mathrm{ha}^{-1}\right)$, Xylopia brasiliensis $\left(4,04 \mathrm{~m}^{3} \mathrm{ha}^{-1}\right)$, Rollinia silvatica $\left(3,54 \mathrm{~m}^{3} \mathrm{ha}^{-1}\right)$ e Miconia cinnamomifolia $\left(3,25 \mathrm{~m}^{3} \mathrm{ha}^{-1}\right)$. $\mathrm{Na}$ área de reserva legal, verificou-se um estoque de volume total de $54,69 \mathrm{~m}^{3}$ $\mathrm{ha}^{-1}$, onde as cinco espécies seguintes representavam $47,67 \%$ do volume total: Mabea fistulifera $\left(6,90 \mathrm{~m}^{3} \mathrm{ha}^{-}\right.$ $\left.{ }^{1}\right)$, Xylopia brasiliensis $\left(6,29 \mathrm{~m}^{3} \mathrm{ha}^{-1}\right)$, Rollinia silvatica $\left(5,08 \mathrm{~m}^{3} \mathrm{ha}^{-1}\right)$, Casearia decandra $\left(4,15 \mathrm{~m}^{3} \mathrm{ha}^{-1}\right)$ e Miconia cinnamomifolia $\left(3,65 \mathrm{~m}^{3} \mathrm{ha}^{-1}\right)$.

R. Árvore, Viçosa-MG, v.31, n.5, p.867-877, 2007 
No $\mathrm{PMF}_{30}$ a área de manejo florestal apresentou um estoque de volume total com casca de $53,02 \mathrm{~m}^{3} \mathrm{ha}^{-1}$, onde as cinco espécies seguintes representaram $57,01 \%$ do volume total: Mabea fistulifera $\left(19,45 \mathrm{~m}^{3} \mathrm{ha}^{-1}\right)$, Apuleia leiocarpa $\left(4,46 \mathrm{~m}^{3} \mathrm{ha}^{-1}\right)$, Matayba elaeagnoides $\left(4,19 \mathrm{~m}^{3} \mathrm{ha}^{-1}\right)$, Xylopia sericea $\left(3,49 \mathrm{~m}^{3} \mathrm{ha}^{-1}\right)$ e Platymenia foliolosa $\left(2,13 \mathrm{~m}^{3} \mathrm{ha}^{-1}\right)$. Na área de reserva legal, observouse um estoque de volume total de $46,91 \mathrm{~m}^{3} \mathrm{ha}^{-1}$, onde as cinco espécies seguintes representaram 52,16\% do volume total: Xylopia sericea $\left(11,31 \mathrm{~m}^{3} \mathrm{ha}^{-1}\right)$, Apuleia leiocarpa $\left(4,45 \mathrm{~m}^{3} \mathrm{ha}^{-1}\right)$, Platymenia foliolosa $\left(4,05 \mathrm{~m}^{3} \mathrm{ha}^{-1}\right)$, Pitptocarpha macropoda $\left(2,45 \mathrm{~m}^{3} \mathrm{ha}^{-1}\right)$ e Matayba elaeagnoides $\left(2,21 \mathrm{~m}^{3} \mathrm{ha}^{-1}\right)$.

No $\mathrm{PMF}_{16}$, a área de manejo florestal apresentou um estoque de volume total com casca de $102,61 \mathrm{~m}^{3}$ ha${ }^{1}$, onde as cinco espécies seguintes representaram $37,48 \%$ do volume total: Croton floribundus $\left(10,02 \mathrm{~m}^{3} \mathrm{ha}^{-1}\right)$, Cecropia glaziovi $\left.8,73 \mathrm{~m}^{3} \mathrm{ha}^{-1}\right)$, Pitptocarpha macropoda $\left(7,00 \mathrm{~m}^{3} \mathrm{ha}^{-1}\right)$, Mabea fistulifera $\left(6,87 \mathrm{~m}^{3} \mathrm{ha}^{-1}\right) \mathrm{e}$ Platypodium elegans $\left(5,66 \mathrm{~m}^{3} \mathrm{ha}^{-1}\right)$. Na área de reserva legal, observouse um estoque de volume total de $95,04 \mathrm{~m}^{3} \mathrm{ha}^{-1}$, onde as cinco espécies seguintes representavam $41,77 \%$ do volume total: Xylopia brasiliensis $\left(14,66 \mathrm{~m}^{3} \mathrm{ha}^{-1}\right)$, Platypodium elegans $\left(8,55 \mathrm{~m}^{3} \mathrm{ha}^{-1}\right)$, Pseudopiptadenia contorta $\left(7,65 \mathrm{~m}^{3} \mathrm{ha}^{-1}\right)$, Dalbergia nigra $\left.4,57 \mathrm{~m}^{3} \mathrm{ha}^{-1}\right) \mathrm{e}$ Anadenanthera peregrina $\left(4,27 \mathrm{~m}^{3} \mathrm{ha}^{-1}\right)$.

Decorrido o tempo de exploração florestal dos PMFs o verificador volume total com casca indicaram que os estoques de volume total das áreas de manejo florestal eram iguais, estatisticamente $(\mathrm{P}>0,05)$, às áreas de reserva legal no $\mathrm{PMF}_{28}$, $\mathrm{PMF}_{30}$ e $\mathrm{PMF}_{16}$ e diferentes $(\mathrm{P}=0,05)$ no $\mathrm{PMF}_{29}$.

A exploração florestal, ao diminuir a densidade absoluta, ativa e acelera os processos de dinâmica sucessional, fazendo que as áreas exploradas evoluam no decorrer do tempo em direção às áreas não-exploradas (SOUZA et al., 1998; TABARELLI, 1997). Gomes et al., (2004), estudando fragmento de floresta de um plano de manejo florestal, após seis anos da exploração florestal, não encontrou diferenças significativas entre as áreas exploradas e as não-exploradas.

\subsection{Alterações na estrutura diamétrica dos PMFs}

\subsubsection{Distribuição de densidade}

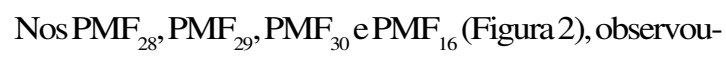
se que, nas áreas de manejo florestal e de reserva legal, a distribuição do número de indivíduos por hectare e por classe de diâmetro seguia o padrão característico de "J-invertido" das florestas ineqüiâneas (MEYER, 1952).
No $\mathrm{PMF}_{28}$, na área de manejo florestal as espécies seguintes ocorreram em todas as classes de diâmetro: Anadenanthera peregrina, Apuleia leiocarpa, Morta, Piptadenia gonoacantha, Carpotroche brasiliensis, Cecropia glaziovi, Duguetia sp., Inga sp., Myrcia fallax, Nectandra oppositifolia. $\mathrm{Na}$ área de reserva legal, as espécies seguintes ocorreram em todas as classes de diâmtro: Ocotea sp., Casearia decandra, Xylopia brasiliensis, Morta, Tachigali multijuga, Tapirira guianensis, Trattinnickia rhoifolia, indeterminada, Inga sp. e Machaerium brasiliense.

No $\mathrm{PMF}_{29}$, na área de manejo florestal as espécies seguintes ocorreram em todas as classes de diâmetro: Casearia decandra, Xylopia brasiliensis, Apuleia leiocarpa, Erythroxylum pelleterianum, Guatteria nigrescens, Lacistema pubescens, Mabea fistulifera, Maprounea guianensis, Miconia cinnamomifolia $\mathrm{e}$ Morta. Na área de reserva legal, as espécies seguintes ocorreram em todas as classes de diâmtro: Rollinia silvatica, Casearia decandra, Lacistema pubescens, Mabea fistulifera, Miconia cinnamomifolia, Morta, Tachigali multijuga, Tapirira guianensis, Trattinnickia rhoifolia e Xylopia brasiliensis.

No $\mathrm{PMF}_{30}$, na área de manejo florestal as espécies seguintes ocorreram em todas as classes de diâmetro: Apuleia leiocarpa, Mabea fistulifera, Matayba elaeagnoides, Platymenia foliolosa, Cecropia glaziovi, Cecropia hololeuca, Croton floribundus, Dictyoloma vandellianum, Guarea guidonia e Hyptidendron asperrimum. $\mathrm{Na}$ área de reserva legal, as espécies seguintes ocorreram em todas as classes de diâmtro: Apuleia leiocarpa, Platymenia foliolosa, Albizia polycephala, Anadenanthera peregrina, Tabernaemontana fuchsiaefolia, Inga edulis, Inga sp., Lacistema pubescens, Matayba elaeagnoides e morta.

No $\mathrm{PMF}_{16}$, área de manejo florestal, as espécies seguintes ocorreram em todas as classes de diâmetro: Platypodium elegans, Apuleia leiocarpa, Croton floribundus, Piptadenia gonoacantha, Xylopia brasiliensis, Anadenanthera peregrina, Cecropia glaziovi, Melanoxylon brauna, Morta e Pitptocarpha macropoda. $\mathrm{Na}$ área de reserva legal, as espécies seguintes apareceram em todas as classes de diâmtro: Platypodium elegans, Dalbergia nigra, Morta, Pseudopiptadenia contorta, Xylopia brasiliensis, Anadenanthera peregrina, Schefflera morototoni, Casearia decandra, Casearia ulmifolia e indeterminada. 

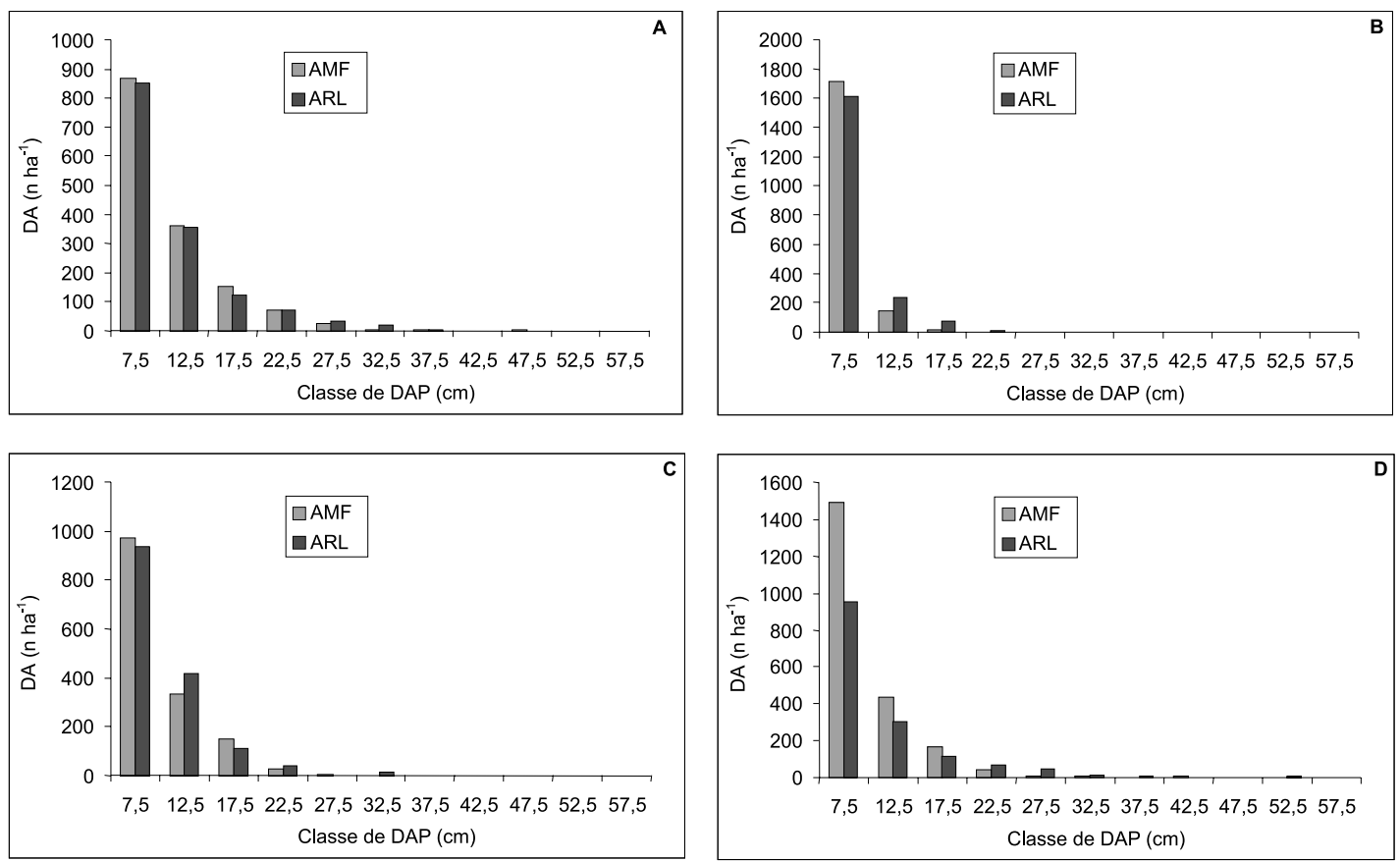

Figura 2 - Distribuição do número de indivíduos por hectare e por classe diamétrica (DA), em áreas de manejo florestal e áreas de reserva legal, dos $\mathrm{PMF}_{28}(\mathrm{~A}), \mathrm{PMF}_{29}(\mathrm{~B}), \mathrm{PMF}_{30}(\mathrm{C})$ e $\mathrm{PMF}_{16}(\mathrm{D})$, nos Municípios de Porto Firme e Rio Espera, Minas Gerais em 2005.

Figure 2 -Distribution of the number of individuals per hectare and per diametric,class (DA), in forest management areas and legal reserve, of the $F M P_{28}(A), F M P_{29}(B), F M P_{30}(C)$ and $F M P_{16}(D)$, in the municipalities of Porto Firme and Rio Espera, Minas Gerais State, in 2005.

\subsubsection{Distribuição da área basal}

No $\mathrm{PMF}_{28}, \mathrm{PMF}_{29}, \mathrm{PMF}_{30}$ e $\mathrm{PMF}_{28}$, as áreas de manejo florestal apresentaram, respectivamente, áreas basais de 14,283 $\mathrm{m}^{2} \mathrm{ha}^{-1}, 7,428 \mathrm{~m}^{2} \mathrm{ha}^{-1}, 9,996 \mathrm{~m}^{2} \mathrm{ha}^{-1} \mathrm{e}$ $16,677 \mathrm{~m}^{2} \mathrm{ha}^{-1} \mathrm{e}$ as áreas de reserva legal, 17,562 $\mathrm{m}^{2}$ ha $^{-1} 10,166 \mathrm{~m}^{2} \mathrm{ha}^{-1}, 9,875 \mathrm{~m}^{2} \mathrm{ha}^{-1}$ e $17,407 \mathrm{~m}^{2} \mathrm{ha}^{-1}$, respectivamente (Figura 3 ).

\subsubsection{Distribuição do volume total com casca}

No $\mathrm{PMF}_{28}, \mathrm{PMF}_{29}, \mathrm{PMF}_{30}$ e $\mathrm{PMF}_{28}$, as áreas de manejo florestal apresentaram, respectivamente, volumes totais com casca de 94,72 $\mathrm{m}^{3} \mathrm{ha}^{-1}, 37,08 \mathrm{~m}^{3} \mathrm{ha}^{-1}$,

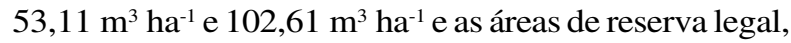
$115,67 \mathrm{~m}^{3}$ ha $^{-1} 54,69 \mathrm{~m}^{3} \mathrm{ha}^{-1}, 49,61 \mathrm{~m}^{3} \mathrm{ha}^{-1}$ e 95,02 $\mathrm{m}^{3}$ ha $^{-1}$, respectivamente (Figura 4 ).

Decorrido o tempo de exploração de cada plano de manejo florestal, os verificadores distribuição da densidade absoluta, área basal e volume total com casca, por hectare e por classe diamétrica, indicaram que as estruturas diamétricas das áreas de manejo florestal são iguais, estatisticamente $(\mathrm{P}>0,05$, ) às áreas de reserva legal no $\mathrm{PMF}_{28}, \mathrm{PMF}_{29}$ e $\mathrm{PMF}_{30}$ e diferentes $(\mathrm{P}=0,05)$ no $\mathrm{PMF}_{16}$.

\subsection{Alterações na estrutura interna dos PMF}

\subsubsection{Infestação de cipós}

Os resultados da avaliação dos PMF, quanto à infestação de cipós, encontram-se no Quadro 2.

As áreas de manejo florestal dos $\mathrm{PMF}_{28}, \mathrm{PMF}_{29}$, $\mathrm{PMF}_{30}$ e $\mathrm{PMF}_{16}$ apresentaram, respectivamente, 66,40\%, $29,68 \%, 58,33 \%$ e $58,19 \%$ do número de indivíduos por hectare, com infestação de cipós. As áreas de reserva legal dos referidos PMFs apresentaram, respectivamente, $38,69 \%, 31,04 \%, 63,86 \%$ e $62,73 \%$ do número de indivíduos por hectare com infestação de cipós. Portanto, ocorreram maiores infestações nas áreas de manejo florestal do $\mathrm{PMF}_{28}$ e naquelas de reserva legal do $\mathrm{PMF}_{29}$, $\mathrm{PMF}_{30} \mathrm{ePMF}_{16}$.

R. Árvore, Viçosa-MG, v.31, n.5, p.867-877, 2007 

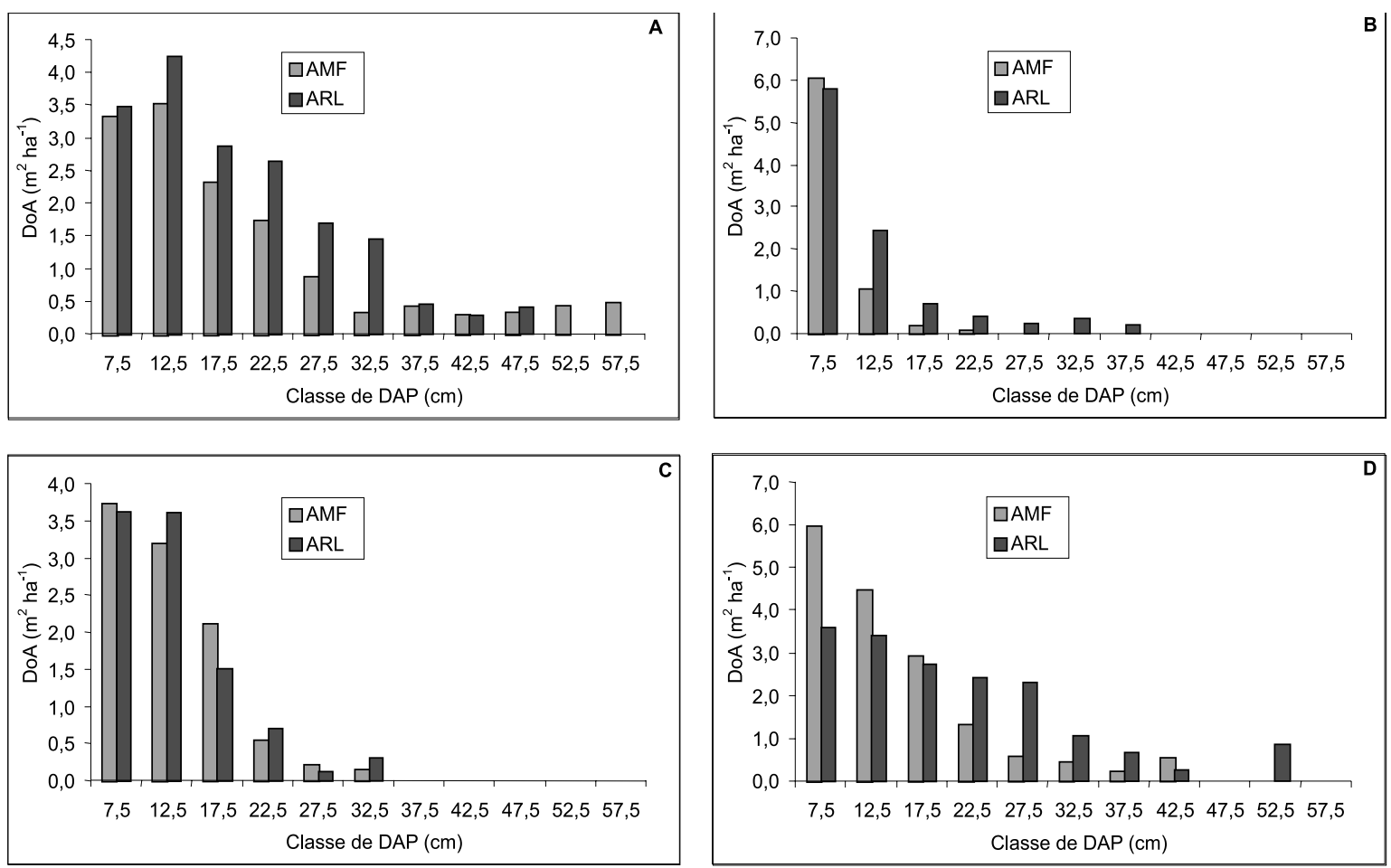

Figura 3 - Distribuição da área basal por hectare e por classe diamétrica (DoA), em área de manejo florestal e área de reserva legal, $\operatorname{dos} \mathrm{PMF}_{28}$ (A), $\mathrm{PMF}_{29}(\mathrm{~B}), \mathrm{PMF}_{30}$ (C) e $\mathrm{PMF}_{16}$ (D), nos Municípios de Porto Firme e Rio Espera, Minas Gerais, em 2005.

Figure 3 - Distribution of the basal area per hectare and per diametric class (DoA), in forest management areas and legal reserve area, of the $F M P_{28}(A), F M P_{29}(B), F M P_{30}(C)$ and $F M P_{16}(D)$, in the municipalities of Porto Firme and Rio Espera, Minas Gerais State, in 2005.

Decorrido o tempo de exploração de cada plano de manejo florestal, o verificador infestação de cipós apresentou, entre as áreas de manejo florestal e as de reserva legal para $\mathrm{PMF}_{29}, \mathrm{PMF}_{30}$ e $\mathrm{PMF}_{16}$, infestação significativamente igual $(\mathrm{P}>0,05)$ e significativamente maior $(\mathrm{P} \leq 0,05)$ no $\mathrm{PMF}_{28}$.

O grau de infestação de cipós é um parâmetro que serve para diagnosticar o estado de conservação de um fragmento florestal suscetível aos efeitos de borda, exploração seletiva de madeira ou lenha e outras formas de interferências antrópicas (SOUZA, 2003). De acordo com Budowski (1996), os cipós são mais abundantes nos primeiros estágios sucessionais, diminuindo sua incidência à medida que a floresta tende a estágios mais avançados. Gomes et al. (2004), estudando o manejo de fragmentos florestais no Município de Matias Barbosa, Minas Gerais, observou uma alta incidência de cipós, tanto na área explorada quanto na não-explorada. O mesmo resultado foi também observado por Almeida e Souza (1997), em estudos realizados na mesma propriedade. A abertura de clareiras, principalmente com interferência antrópica, pode aumentar a incidência de cipós numa área florestal.

\subsubsection{Qualidade de fuste}

Verificou-se (Quadro 3) no $\mathrm{PMF}_{28}$ e $\mathrm{PMF}_{29}$ que, nas áreas de manejo florestal e de reserva legal, a qualidade de fuste 1 (bom) superou as classes 2 e 3 (regular e inferior, respectivamente), indicando maior valor comercial do estoque remanescente. No $\mathrm{PMF}_{30}$ e PMF ${ }_{16}$, observou-se o contrário, ou seja, as classes 2 de qualidade de fuste e 3 (regular e inferior) superaram a classe 1 (bom), indicando menor valor comercial do estoque remanescente das áreas manejadas.

Decorrido o tempo de execução dos planos de manejo florestal, o verificador qualidade de fuste mostrou diferenças não-significativas $(\mathrm{P}>0,05)$ entre as áreas de manejo florestal e de reserva legal de todos os planos de manejo florestal. 

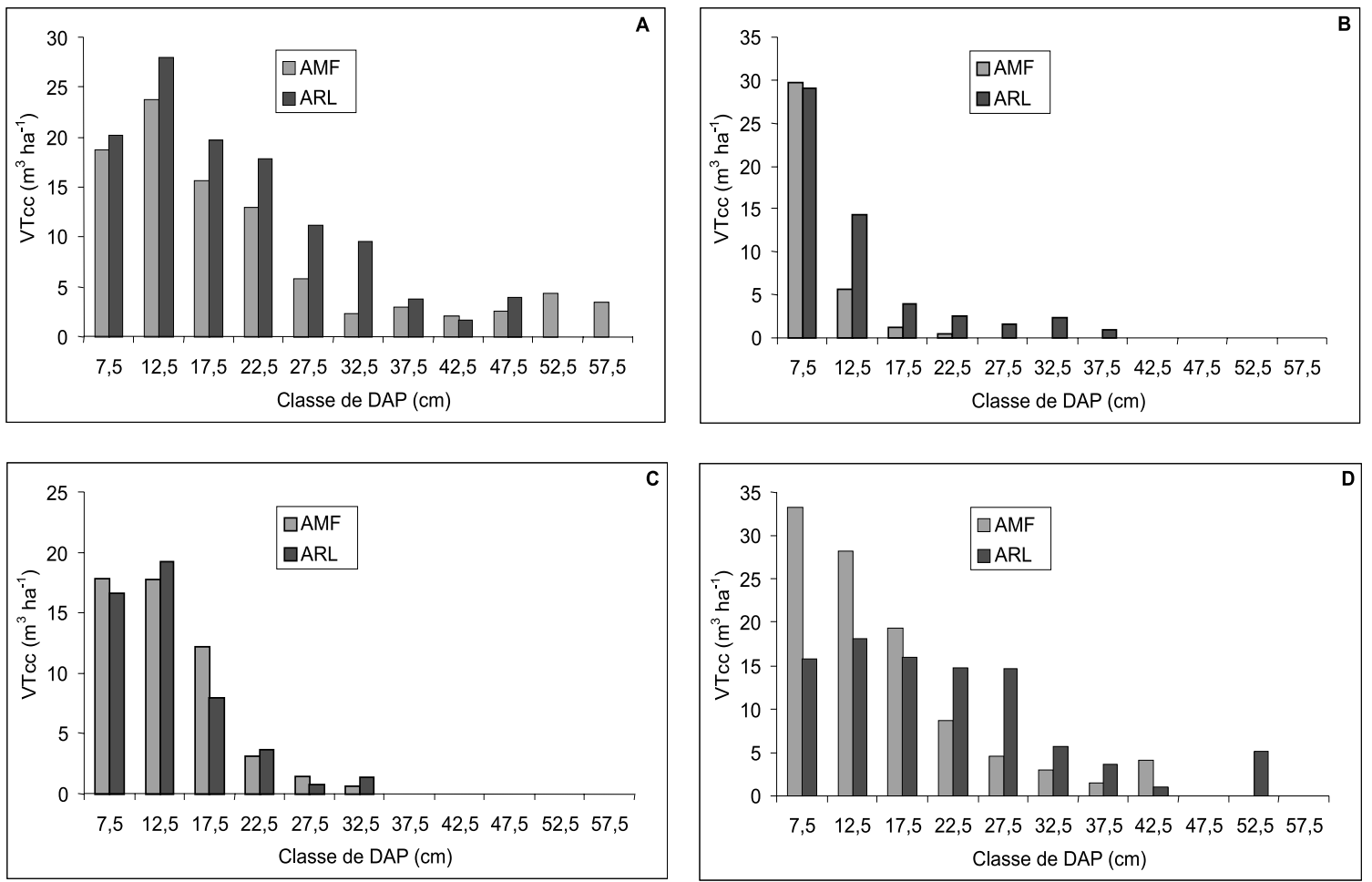

Figura 4 - Distribuição do volume total com casca por hectare e por classe diamétrica (VTcc), em áreas de manejo florestal e áreas de reserva legal, dos $\mathrm{PMF}_{28}(\mathrm{~A}), \mathrm{PMF}_{29}(\mathrm{~B}), \mathrm{PMF}_{30}(\mathrm{C})$ e $\mathrm{PMF}_{16}(\mathrm{D})$, nos Municípios de Porto Firme e Rio Espera, Minas Gerais.

Figure 4 -Distribution of the outside bark volume per hectare and per diametric class (VTcc, in forest management areas and legal reserve area, of the $F M P_{28}(A), F M P_{29}(B), F M P_{30}(C)$ and $F M P_{16}(D)$, in the municipalities of Porto Firme and Rio Espera, Minas Gerais State, in 2005.

Quadro 2 - Distribuição do número de indivíduos por hectare das espécies arbóreas, por infestação de cipós, em áreas de manejo florestal e áreas de reserva legal dos $\mathrm{PMF}_{28}, \mathrm{PMF}_{29}, \mathrm{PMF}_{30}$ e $\mathrm{PMF}_{16}$, nos Municípios de Porto Firme e Rio Espera, Minas Gerais, em 2005

Table 2 - Distribution of the number of individuals per hectare of tree species by infestation by lianes in forest management areas and legal reserve areas for $\mathrm{PMF}_{28}, \mathrm{PMF}_{29}, \mathrm{PMF}_{30}$ and $\mathrm{PMF}_{16}$, in the municipalities of Porto Firme and Rio Espera, Minas Gerais State, in 2005

\begin{tabular}{|c|c|c|c|c|c|c|c|c|}
\hline \multirow{4}{*}{ Característica } & \multicolumn{8}{|c|}{ Número de Indivíduos por Hectare $\left(\mathrm{n} \mathrm{ha}^{-1}\right)$} \\
\hline & \multicolumn{8}{|c|}{ Condições de Floresta } \\
\hline & \multicolumn{4}{|c|}{$\overline{\mathrm{AMF}}$} & \multicolumn{4}{|c|}{ ARL } \\
\hline & $\mathrm{PMF}_{28}$ & $\mathrm{PMF}_{29}$ & $\mathrm{PMF}_{30}$ & $\mathrm{PMF}_{16}$ & $\mathrm{PMF}_{28}$ & $\mathrm{PMF}_{29}$ & $\mathrm{PMF}_{30}$ & $\mathrm{PMF}_{16}$ \\
\hline Árvore sem cipó & 504 & 1322 & 620 & 904 & 900 & 1342 & 552 & $\overline{562}$ \\
\hline Árvore com cipó no tronco & 248 & 312 & 246 & 370 & 256 & 380 & 222 & 236 \\
\hline Árvore com cipó na copa & 334 & 116 & 222 & 346 & 180 & 92 & 356 & 228 \\
\hline Árv. cipó tronco e na copa & 414 & 130 & 400 & 542 & 132 & 132 & 394 & 482 \\
\hline Total & $1.500^{*}$ & $1.880^{\mathrm{ns}}$ & $1.488^{\mathrm{ns}}$ & $2.162^{\mathrm{ns}}$ & 1.468 & 1.946 & 1.524 & 1.508 \\
\hline
\end{tabular}

* Significativo a $5 \%$ e ns: não-significativo a $5 \%$. 
Quadro 3 - Distribuição do número de indivíduos por qualidade de fuste das espécies arbóreas, em áreas de manejo florestal e áreas de reserva legal, dos $\mathrm{PMF}_{28}, \mathrm{PMF}_{29}, \mathrm{PMF}_{30}$ e $\mathrm{PMF}_{16}$, nos Municípios de Porto Firme e Rio Espera, Minas Gerais

Quadro 3 -Distribution of the number of individuals per log quality of tree species, in forest management areas and legal reserve areas for $P M F_{28}, P M F_{29}, P M F_{30}$ and $P M F_{10}$ in the municipalities of Porto Firme and Rio Espera, Minas Gerais State, in 2005

\begin{tabular}{|c|c|c|c|c|c|c|c|c|}
\hline \multirow[t]{4}{*}{ Qualidade de Fuste } & \multicolumn{8}{|c|}{ Número de indivíduos por hectare } \\
\hline & \multicolumn{8}{|c|}{ Condições de Floresta } \\
\hline & \multicolumn{4}{|c|}{ AMF. } & \multicolumn{4}{|c|}{$\mathrm{ARL}$} \\
\hline & $\mathrm{PMF}_{28}$ & $\mathrm{PMF}_{29}$ & $\mathrm{PMF}_{30}$ & $\mathrm{PMF}_{16}$ & $\mathrm{PMF}_{28}$ & $\mathrm{PMF}_{29}$ & $\mathrm{PMF}_{30}$ & $\mathrm{PMF}_{16}$ \\
\hline 1-Bom $(80$ a $100 \%)$ & 810 & 1.114 & 712 & 1.044 & 818 & 1.144 & 776 & 612 \\
\hline 2 -Regular (50 a $79 \%$ ) & 572 & 676 & 616 & 792 & 536 & 658 & 498 & 638 \\
\hline 3-Inferior $(<50 \%)$ & 118 & 90 & 160 & 326 & 114 & 144 & 250 & 258 \\
\hline Total & $1.500^{\mathrm{ns}}$ & $1.880^{\mathrm{ns}}$ & $1.488^{\mathrm{ns}}$ & $2.162^{\mathrm{ns}}$ & 1.468 & 1.946 & 1.524 & 1.508 \\
\hline
\end{tabular}

ns: não-significativo a $5 \%$, pelo teste $\mathrm{t}$.

\section{CONCLUSÕES}

Os resultados deste estudo permitiram extrair as seguintes conclusões gerais decorridos 8 anos das atividades de exploração florestal no $\mathrm{PMF}_{29}, 9$ anos no $\mathrm{PMF}_{30}, 10$ anos no $\mathrm{PMF}_{28}$ e 11 anos no $\mathrm{PMF}_{16}$

\subsection{Alterações na estrutura horizontal dos PMFs}

$O$ verificador densidade absoluta indicou que as áreas de manejo florestal eram iguais, estatisticamente $(\mathrm{P}>0,05)$, às áreas de reserva legal no $\mathrm{PMF}_{28}, \mathrm{PMF}_{29}$ e $\mathrm{PMF}_{30}$ e diferentes, estatisticamente $(\mathrm{P} \leq 0,05)$ no $\mathrm{PMF}_{16}$.

Os verificadores dominância absoluta e volume total com casca apontaram que os estoques de área basal e volume total das áreas de manejo florestal eram iguais, estatisticamente $(\mathrm{P}>0,05)$, às áreas de reserva legal no $\mathrm{PMF}_{28}, \mathrm{PMF}_{30}$ e $\mathrm{PMF}_{16}$ e diferentes, estatisticamente $(\mathrm{P} \leq 0,05)$, no $\mathrm{PMF}_{29}$.

\subsection{Alterações na estrutura diamétrica dos PMFs}

Os verificadores distribuição da densidade absoluta, área basal e volume total com casca, por hectare e por classe diamétrica, indicaram que as estruturas diamétricas das áreas de manejo florestal são iguais, estatisticamente $(\mathrm{P}>0,05)$, às áreas de reserva legal nos $\mathrm{PMF}_{28}, \mathrm{PMF}_{29}$, e $\mathrm{PMF}_{30}$ e diferentes, estatisticamente $(\mathrm{P} \leq 0,05)$, no $\mathrm{PMF}_{16}$.

\subsection{Alterações na estrutura interna dos PMFs}

O verificador infestação de cipós indicou que as áreas de manejo florestal possuíam infestação de cipós igual, estatisticamente $(\mathrm{P}>0,05)$, às áreas de reserva legal no $\mathrm{PMF}_{29}, \mathrm{PMF}_{30}$ e $\mathrm{PMF}_{16}$ e diferentes, estatisticamente $(\mathrm{P} \leq 0,05)$, no $\mathrm{PMF}_{28}$.

R. Árvore, Viçosa-MG, v.31, n.5, p.867-877, 2007
$\mathrm{O}$ verificador qualidade de fuste mostrou que as áreas de manejo florestal possuíam qualidade de fuste igual, estatisticamente $(\mathrm{P}>0,05)$, às áreas de reserva legal em todos os PMFS.

\section{REFERÊNCIAS}

ALMEIDA, D. S.; SOUZA, A. L. Florística e estrutura de um fragmento de floresta atlântica, no município de Juiz de Fora, Minas Gerais.

Revista Árvore, v.21, n.2, p.221-230, 1997.

MINAS GERAIS - ASSEMBLÉIALEGISLATIVADO ESTADO Caracterização dos municípios mineiros com base em informações do IGA-CETEC e IBGE. Disponível em: <www.almg.gov.br>. Acesso em: 7 dez. 2005.

BUDOWSKI, G. Los bosques de los trópicos húmedos de América. Turrialba, v.16, n.3, p.278-285, 1966.

CIENTEC 2006. Mata Nativa 2.: sistema para análise fitossociológica e elaboração de inventários e planos de manejo de florestas nativas. Versão,2. Viçosa, MG, Cientec Consultoria e Desenvolvimento de Sistemas, 2006.

COELHO, D. J. S.; SOUZA, A. L. Modelo de gestão florestal sustentável para microrregião de Viçosa, Minas Gerais. Revista Árvore, v.26, n.2, p.145-154, 2002.

FUNDAÇÃO CENTRO TECNOLÓGICO DE MINAS GERAIS - CETEC. Equações volumétricas aplicáveis ao manejo sustentável de florestas nativas no estado de Minas Gerais. Belo Horizonte: 1995. 295p. 
GOMES, A. P. C.; SOUZA, A. L.; MEIRANETO, J.A. A. Alteração estrutural de uma área florestal explorada convencionalmente na bacia do Paraíba do Sul, Minas Gerais, nos domínios de Floresta Atlântica. Revista Árvore, v.28, n.3, p.407-417, 2004.

INSTITUTO ESTADUAL DE FLORESTAS - IEF. Contrato BIRD/ SEPLAN/IEF-MG. Programa Prófloresta. Cobertura vegetal e uso do solo do Estado de Minas Gerais. Belo Horizonte: 1994. 1 mapa: color. 99x66. Escala: 1:1.500.000. Material cartográfico.

LEITE, H. G.; OLIVEIRA, F. H. T. Statistical procedure to test the identity of analytical methods. Communications in Soil Science and Plant Analysis, v.3, p.7/8, 2002.

LILLIEFORS, H. W. One the kolmogorov-smirnov test for normality with mean and variance unknown. Jornal of the American Statistical Association, v.64, p.399-402, 1967.

MAGURRAN, A. E. Ecological diversity and its measurement. Princeton: Princeton University Press, 1988. 167 p.

MEYER, H. A. Structure, growth, and drain in balanced unven-aged forests. Journal of Forestry, v.50, n.2, p.85-92, 1952.

MUELLER-DOMBOIS, D.; ELLENBERG, H. Aims and methods of vegetation ecology. New York: John Wiley \& Sons, 1974. 547p.
SISTEMA PARAANÁLISES ESTATÍSTICAS SAEG, versão 9.0. Viçosa, MG: Fundação Arthur Bernardes, 2005.

SOUZA, A. L. et al. Avaliação dos impactos das operações de exploração de madeira e de tratamentos silviculturais num projeto de manejo sustentável. Viçosa, MG: Universidade Federal de Viçosa, 1998. 248p. (Relatório Técnico de Pesquisa-CNPq. Processo 520010/96-5).

SOUZA, A. L. et al. Dinâmica da regeneração natural de uma floresta ombrófila densa secundária, após corte de cipós, reserva natural da Companhia Vale do Rio Doce S.A., Estado do Espírito Santo, Brasil. Revista Árvore, v.26, n.4, p.411-419, 2003.

TABARELli, M. A Regeneração da floresta atlântica montana. 1997. 104 f. Tese (Doutorado em Engenharia Florestal) Universidade de São Paulo, São Paulo, 1997.

VELOSO, H. P. Manual técnico da vegetação brasileira. Rio de Janeiro: IBGE, 1992. 92p. (Série Manuais Técnicos de Geociências, 1).

WINER, B. J. Statistical principles in experimental design. New york: McGrawHill, 1962. 94p. 